\title{
Angular control of a hybrid magnetic metamolecule using anisotropic FeCo
}

\author{
S. A. Gregory,${ }^{1}$ L. C. Maple, ${ }^{1}$ G. B. G. Stenning, ${ }^{2}$ T. Hesjedal,${ }^{3}$ G. van der Laan,${ }^{4}$ and G. J. Bowden ${ }^{1}$ \\ ${ }^{1}$ School of Physics and Astronomy, University of Southampton, SO17 1BJ, United Kingdom \\ ${ }^{2}$ ISIS Neutron and Muon Source, Rutherford Appleton Laboratory, Didcot, OX11 OQX, United Kingdom \\ ${ }^{3}$ Department of Physics, Clarendon Laboratory, University of Oxford, Oxford, OX1 3PU, United Kingdom \\ ${ }^{4}$ Magnetic Spectroscopy Group, Diamond Light Source, Didcot, OX11 ODE, United Kingdom
}

(Dated: September 4, 2015)

\begin{abstract}
By coupling magnetic elements to metamaterials, new hybrid metamolecules can be created with interesting properties such as photo-magnon coupling. Here, we present results for a hybrid metamolecule, using a hard as opposed to soft magnetic material. This was achieved by placing a thin film of single crystal hard FeCo in close proximity to a split ring resonator (SRR). To suppress eddy-current shielding the FeCo film was patterned into $100 \mu \mathrm{m}$ disks. The resulting metamolecule exhibits photon-magnon coupling when the FeCo is on resonance (FMR). Three new features are demonstrated and discussed. One, hard magnets allow FMR to be performed in near zero field, thereby partially eliminating the need for applied fields. Two, because the FMR is anisotropic, angular control over hybrid SRR/FMR resonances is achieved. Three, the single crystal FeCo can be re-magnetized parallel/perpendicular to the plane of the SRR, in real time, thus opening the door to magnetically configurable metamaterials. Finally, the work was performed using coplanar waveguide (CPW) excitation. A study was made therefore of how best to excite the numerous transverse magnetic (TM) and transverse electric (TE) modes of the SRR, using near-field CPWexcitation. Such detail is important, if the strongest signals are to be achieved. Photon-magnon coupling strengths of up to $5 \%$ are demonstrated.
\end{abstract}

\section{INTRODUCTION}

Given the successful development of passive metamaterials with impressive applications, such as negative refractive index, invisibility cloaks and super-lenses ${ }^{1,2}$, there has been a concerted effort in recent years to develop active metamaterials ${ }^{3}$. Such metamaterials can be controlled externally, allowing exploitation of new functionalities in the time domain. For example, active metamaterials have been used $(i)$ to enhance fast non-linear switching $^{3}$, and (ii) to enhance luminescence in plasmonic quantum dot metamaterials ${ }^{4}$. This work, in turn, has stimulated research into tunable hybrid meta-devices which exploit micro-electro-mechanical systems (MEMs), semiconductors, phase change materials, liquid crystals, superconductors and magnetic media ${ }^{5-7}$. In particular, magnetic materials have much to offer in the field of externally controllable non-linear active metamaterials, given their inherent non-linear magnetic behavior.

To date, research on magnetic-hybrid metamaterials has focused, primarily, on coupling ohmic metamaterials, such as copper split-ring resonators (SRR), with the ferromagnetic resonance (FMR) of ferrites ${ }^{8-19}$. In general, this work has been performed largely using magnetically isotropic ferrites such as yttrium iron garnet (YIG), notable for their widespread use in microwave electronics and low-loss magnetic resonance behavior ${ }^{9,18,19}$. In practice, the majority of experiments have been carried out at relatively low frequencies of $\sim 10 \mathrm{GHz}$ using cavity waveguides and bulk YIG single crystals ${ }^{10,14,16}$. An exception to this is the work reported in Refs. 9 and 20 which used coplanar waveguides (CPW) both to excite the SRR and demonstrate magnetic control over the response of the SRR. Such planar device structures lend themselves to applications in microwave electronics ${ }^{21-24}$.

However, YIG and other ferrites are restricted in their application because of their limited frequency range and/or high magnetic field demands. In addition, they are hard either to pattern or incorporate into multilayer structures. By contrast, the development of devices based on patterning metallic films has undergone a revolution over the last 25 years $^{25}$. Metallic magnetic films can be easily patterned to micron or sub-micron length scales and incorporated into bilayers, multilayers or magnetic spin-valve structures. In turn, this allows access to a rich variety of magnetic phenomena such as magnetic exchange bias ${ }^{26}$, spintronics ${ }^{27}$, magnetic vortex states in geometrically confined structures ${ }^{28}$, skyrmions ${ }^{29}$ and the new range of magnonic functionalities ${ }^{30,31}$. Of course, it should be acknowledged at the outset that highfrequency induced eddy-currents in the transition metal films threaten to limit their use in active meta-devices. However, if the transition metal films are patterned into unconnected $100 \mu \mathrm{m}$ disks, issues with eddy-currents can be effectively negated. In particular, giant magnetic control (40 dB) has been demonstrated, over a SRR resonance at $17 \mathrm{GHz}$ coupled to patterned disks of isotropic 'permalloy'20.

In this paper, we present results for a magnetic hybrid molecule, incorporating a hard FeCo film with remanent magnetic states. The thin film of single-crystalline FeCo was grown by molecular beam epitaxy (MBE) and subsequently patterned into $100 \mu \mathrm{m}$ disks. This allows two new features to be demonstrated: $(i)$, hard magnetic materials enables FMR to be performed in zero or near zero applied field, thus partially eliminating the need for strong applied fields; (ii), because the FMR is anisotropic, control over the angle of the hybrid meta- 
molecule is achieved. As before, the advantages of a planar system are still maintained, i.e., device-ready and fully compatible with cleanroom processes ${ }^{20}$. So, in principle, it should be possible to pattern such films for magnonic applications and hard-drive technologies.

Finally, we report a special study of how to excite the various transverse magnetic (TM) and transverse electric (TE) modes of the SRR, using the near-field excitation of the CPW. By sliding a SRR over the transmission lines of the CPW, certain SRR modes are favored while others are suppressed. Such detail is important, if the strongest signal/modulation of a metamolecule resonance is to be achieved. In passing, we also note that physical movement of an SRR over a CPW enables MEMs-like modulation of metamolecule resonances.

\section{EXPERIMENTAL DETAILS}

Much of the hardware used for the experiments reported here has already been described in Refs. 9 and 20. So only a minimalistic description will be given here. A block schematic of the apparatus can be seen in Fig. 1.

The single-crystalline $\mathrm{Fe}_{50} \mathrm{Co}_{50}$ film was grown epitaxially on an $\mathrm{MgO}(001)$ substrate measuring $10 \times 12 \mathrm{~mm}^{2}$. A 3-nm-thick Fe seed layer was used to promote singlecrystal growth of the 30 -nm-thick $\mathrm{Fe}_{50} \mathrm{Co}_{50}$ film. The substrate was subsequently cut up into $5 \times 5 \mathrm{~mm}^{2}$ pieces for the experiment. The MBE growth procedure was identical to that for the FeCo film used in x-ray detected FMR experiments described in Ref. 32. The 30-nm-thick FeCo film was subsequently patterned into $100 \mu \mathrm{m}$ disks, using standard lithographic techniques in the clean-room facilities at the University of Southampton. An $8 \mu \mathrm{m}$ thick insulating SU- $8^{33}$ layer was subsequently spun and cured on top of the FeCo disks. Next, a 300-nm-thick film of copper was deposited on top of the SU-8 layer and patterned into an SRR. Their proximity ensures excellent coupling between the FeCo disks and the SRR. The dimensions of the SRR were identical to those of Ref. 9. Finally, a $1.3 \mu \mathrm{m}$ thin layer of photoresist was deposited on top of the SRR to provide insulation from the CPW. For the experiments, the FeCo/SRR stack was placed 'flip-chip' on top of the CPW, as shown in Fig. 1(a). A schematic diagram of the stack, photograph, and SRR dimensions can be found in Sec. IV.

For the experiments, the CPW was connected to a vector network analyzer (VNA) which provides the microwave excitation while measuring the absorption/transmission properties of the loaded CPW in the form of the scattering matrix $S$. For the magnetic field rotation experiments, the sample was placed in a portable octupole magnet system (POMS) at the Diamond Light Source $^{34}$. This magnet allows fields of up to $0.9 \mathrm{~T}$ to be applied in any direction. Thus microwave transmission and reflection, the parameters $S_{21}$ and $S_{11}$, respectively, can be measured both as a function of applied magnetic (a)

(b)
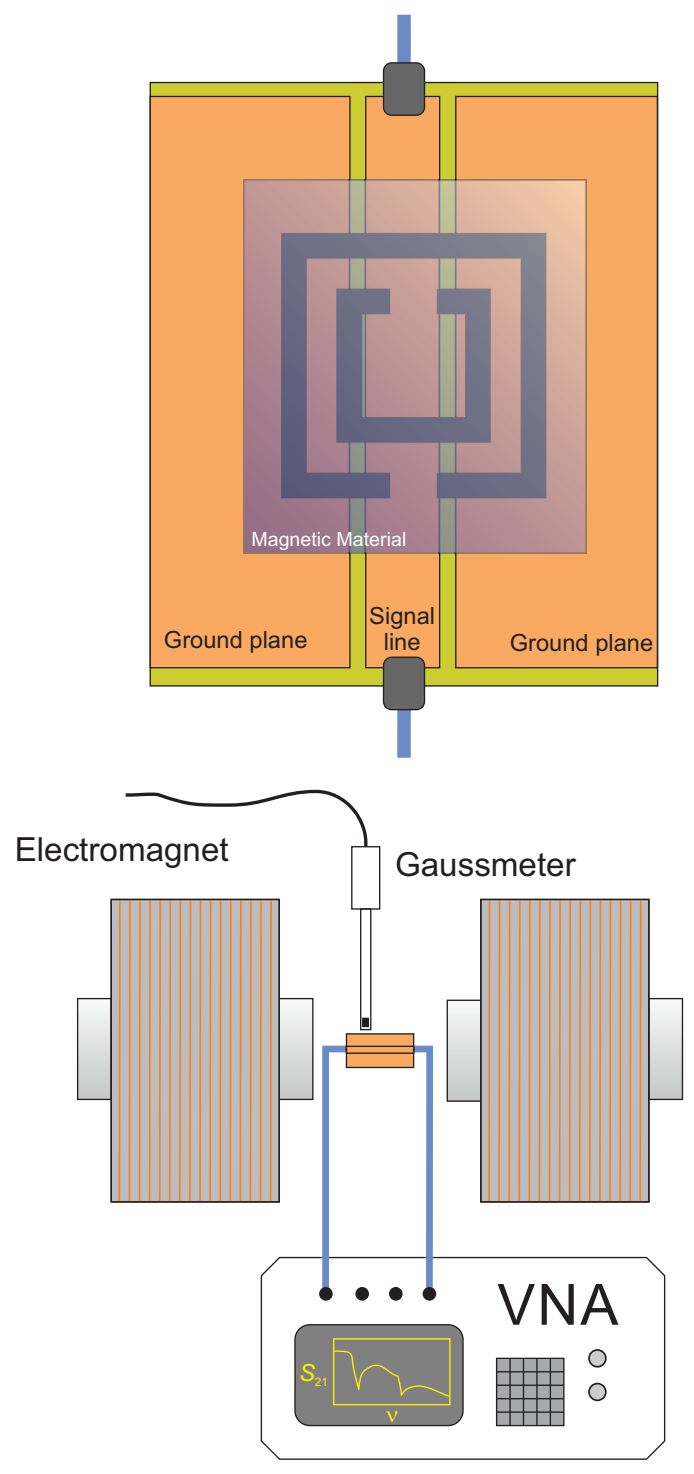

FIG. 1. Schematic diagram of the basic components in the experimental setup. (a) The split ring resonator (SRR, shown in black) is mounted on a grounded plane CPW (yellow/orange). The magnetic FeCo patterned disks (not shown) are atop the SRR. (b) A vector network analyzer (VNA) provides microwaves to the loaded $\mathrm{CPW}$, in the frequency range 1$20 \mathrm{GHz}$. The VNA simultaneously measures the transmitted and reflected signal of the nominally $50 \Omega$ loaded CPW (the $S$-parameters). The electromagnet provides magnetic fields of up to $0.9 \mathrm{~T}$.

field strength and orientation. The former is important, because the FeCo FMR is strongly angle-dependent, due to the four-fold magnetic anisotropy of the FeCo layer in the plane of the film.

Finally, it should be noted that the highly localized microwave-driving fields from the $\mathrm{CPW}$, and mutual coupling between the CPW and SRR, are both strongly dependent on the relative position of the SRR with respect to the CPW transmission lines. Moreover, the SRR resonances are characterized by several TM and TE modes, 
all of which are strongly dependent on the near-field excitation of the CPW. Thus if maximum signal/modulation is required, it is important to know which SRR mode is being excited and how to maximize it. Thus prior to carrying out the experiments reported here, we decided to carry out a special study of the SRR modes of excitation, both experimentally, and with finite element simulations.

\section{EXCITATION OF SRR TE AND TM MODES USING CPW EXCITATION}

Foremost, it is important to appreciate that the strength of the electromagnetic-fields (EM) generated by the CPW fall away very sharply with increasing distance from the CPW transmission lines. To examine this feature, numerical simulations of the 'unloaded' CPW electric and magnetic fields were examined using the finite element package COMSOL. The results obtained at $10 \mathrm{GHz}$ can be seen in Fig. 2(a,b). It is immediately obvious that that the EM energy of the traveling wave is largely confined to the gaps in the transmission lines of the $\mathrm{CPW}$, with a wavelength along the $\mathrm{CPW} \approx(2 / 3) \lambda_{\text {free-space. }}$ In particular, the EM-fields at right-angles to the CPW are highly localized decaying rapidly on a length scale which is much smaller than the SRR size. In essence, therefore FMR experiments performed using CPW-excitation can be described as 'nearfield excitation'31,35. To maximize signal, it is therefore important to place both the SRR and FeCo disks as close as possible to the CPW transmission lines (in practice $\mu \mathrm{m}$ not $\mathrm{mm})$.

Secondly, it is well known that the SRR resonances are characterized by several TE- and TM-modes of excitation $^{36-39}$. For example, in free-space TM polarization excites magnetic modes and TE polarization excites electric modes ${ }^{37}$. Of course, the situation is more complicated using near-field CPW excitation. The EM-fields are highly localized around the signal line and gaps of the CPW. Thus the system is highly spatially sensitive. In principle, it should be possible to simulate the effect of moving the SRR with respect to the CPW. However, in practice this is difficult because $(i)$ it is computationally expensive, (ii) technically challenging, due to large variation in mesh length scales (e.g., CPW length $\approx 3 \mathrm{~cm}$, SRR thickness $\approx 300 \mathrm{~nm}$ ), and, (iii) there are difficulties in accounting for stray capacity. Of course, these disadvantages can be overcome by performing real-time experiments. We decided to physically move an SRR across the CPW transmission lines while simultaneously measuring the $S$-parameters of the system. These translation experiments were performed using a copper SRR patterned onto a glass plate, placed directly on a grounded CPW. The SRR was then pulled at right angles across the CPW using a strip of acetate attached to a micrometer stage. A schematic representation of the experiment can be seen in Fig. 3(a). The resulting $S_{21}$ scans, as a function of translation and frequency, are summarized in Fig. 3(c).
This shows that as the SRR is moved across the CPW transmission lines, several SRR resonances appear and disappear.

The resonances in Fig. 3(c) are labeled $\mathrm{TM}_{o}^{1}, \mathrm{TM}_{i}^{1}$, and $\mathrm{TE}_{o}^{1}$, from left to right. The superscript 1 refers to 'first' or lowest frequency mode. In practice, this 'mode index' is defined by the number of current density nodes around the $\mathrm{SRR}^{37}$. However, the subscript indicates whether the mode is driven primarily by the outer ring $(o)$ or inner ring $(i)$ of the $\mathrm{SRR}^{38,39}$. In addition, our reasons for labeling the resonances in this way are also based on our simulations summarized in Fig. 4(a,b). These were obtained using finite element simulations of a 2D infinite metamaterial of square SRRs sandwiched between FR4 ${ }^{40}$ and glass. The SRR dimensions were identical to those used in the experiments, except that the thickness of the SRR was set at $0.3 \mathrm{~mm}$, rather than $300 \mathrm{~nm}$, to aid meshing. These computational results allow us to identify the nature of SRR mode spectrum [see Fig. 4(a)], and take 'snapshots' of the individual modes versus frequency [see Fig. 4(b)].

In practice, both the inner- and outer-ring excitations of the SRR resonances can be identified, with the latter occurring at a lower frequency ${ }^{38,39}$. It follows therefore that the lowest frequency mode appearing at $\sim 7 \mathrm{GHz}$ in Fig. 3(c) is the outer ring driven magnetic mode $\mathrm{TM}_{o}^{1}$. The next two modes, appearing between 10 and $18 \mathrm{GHz}$, are the inner ring driven magnetic mode $\mathrm{TM}_{i}^{1}$ and outer ring driven electric mode $\mathrm{TE}_{o}^{1}$, respectively. By symmetry, only TE modes are excited when the SRR is placed symmetrically over the center of the CPW, while the asymmetric TM modes are suppressed. These features are clearly evident in the experimental results presented in Fig. 3(b). Furthermore, the outer ring modes appear earlier and disappear later than the inner ring modes, because they are first over the CPW signal line and the last to leave.

Finally, by using a grounded CPW rather than an ungrounded one, the (raw) transmission properties are improved and unwanted CPW resonances are suppressed.

\section{FeCo FMR RESULTS}

As mentioned in Sec. II, the CoFe film was patterned into $100 \mu \mathrm{m}$ disks to suppress eddy currents. Disks were chosen to negate any shape anisotropy in the plane of the film. Many more details of the $\mathrm{CoFe} / \mathrm{SRR}$ stack, and its placement on the CPW can be seen in Fig. 5(a-c).

For the experiments the SRR was placed on the CPW with its center at about $1 \mathrm{~mm}$ from the center of the CPW to favor excitation of both $\mathrm{TM}_{o}^{1}$ and $\mathrm{TE}_{o}^{1}$ modes. Once in position, the sample was fixed to the CPW using PTFE tape. The loaded CPW, connected to the VNA, was subsequently placed within the octupole magnet. Prior to any measurements, the FeCo was saturated in a field of $0.3 \mathrm{~T}$, in the desired direction, to ensure well-defined magnetic states. Also during the experiments, the CPW 
(a)
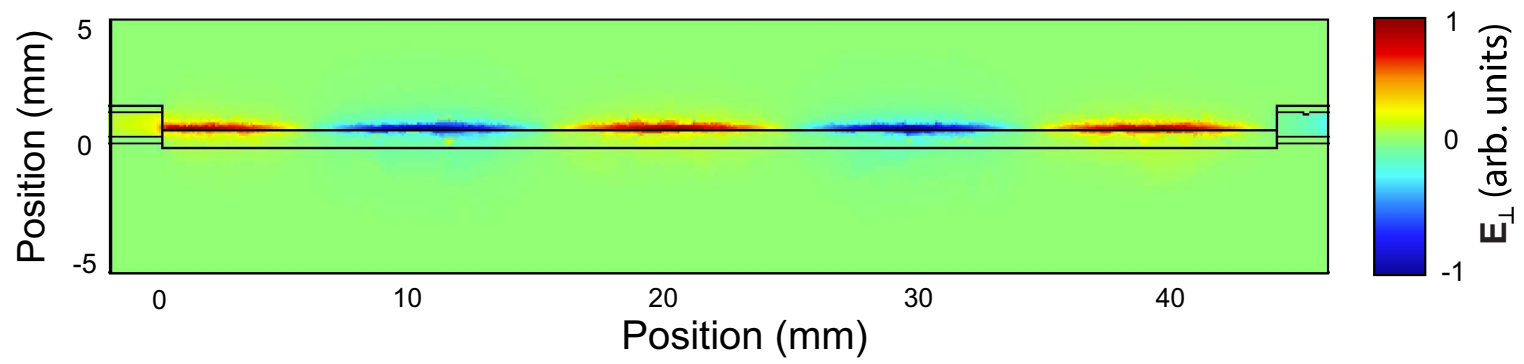

(b)

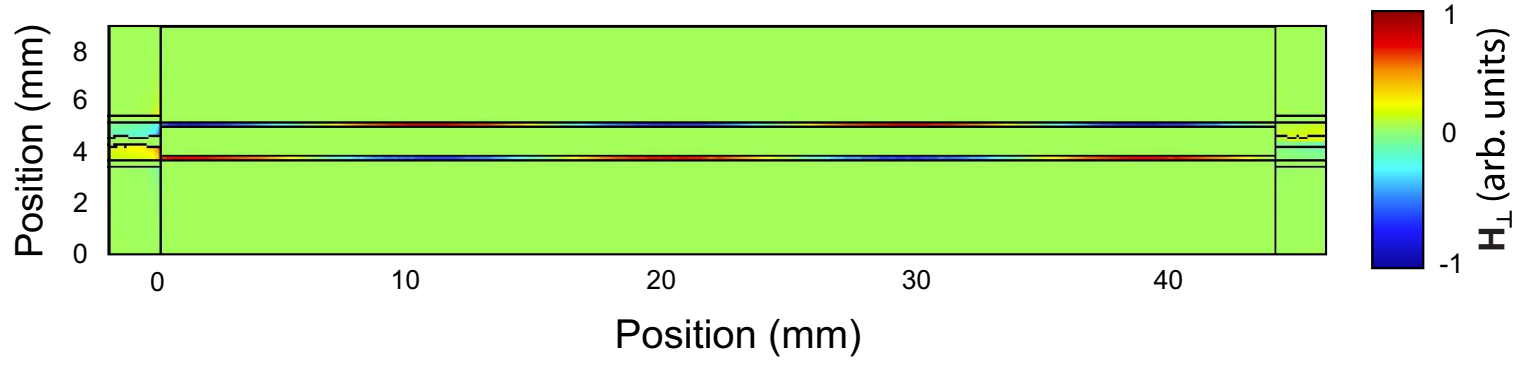

FIG. 2. Computer simulation, using COMSOL, of the electric and magnetic field distributions in the CPW. (a) Side-view of the horizontal component of the electric field ( $E$-field), and, (b) view from above of the vertical component of magnetic field ( $H$-field).

and microwave cables were not moved since any shift in cable position and/or connections alters the transmission/reflection properties measured by the VNA. The raw transmission $S_{21}$ of the loaded CPW, as a function of frequency, in zero field, can be seen in Fig. 6.

With this experimental arrangement, it is an easy matter to determine the FMR of the FeCo disks as the magnetic field is rotated in the plane of the FeCo film. The results obtained in this way are summarized in Fig. 7 .

The FMR data can be fitted using the Kittel equations ${ }^{41,42}$. Explicitly:

$$
\begin{aligned}
\nu & =\gamma \sqrt{\mu_{0} H_{\mathrm{eff}} B_{\mathrm{eff}}} \\
H_{\mathrm{eff}} & =H \cos \left(\phi_{M}-\phi_{H}\right)+\frac{2 K_{C}}{\mu_{0} M_{0}} \cos 4\left(\phi_{M}-\phi_{C}\right) \\
& -\frac{2 K_{U}}{\mu_{0} M_{0}} \cos 2\left(\phi_{M}-\phi_{U}\right) \\
B_{\mathrm{eff}} & =\mu_{0} H \cos \left(\phi_{M}-\phi_{H}\right)+\mu_{0} M_{0} \\
& +\frac{K_{C}}{2 M_{0}}\left(3+\cos 4\left(\phi_{M}-\phi_{C}\right)\right) \\
& -\frac{2 K_{U}}{M_{0}}\left(1+\cos 2\left(\phi_{M}-\phi_{U}\right)\right)
\end{aligned}
$$

Here, $\nu$ is the frequency, $K_{U}$ and $K_{C}$ are uniaxial and cubic anisotropy terms, while $\phi_{M} ; \phi_{H}, \phi_{U}$, and $\phi_{C}$ are the in-plane angles of magnetization, applied field, uniaxial and cubic easy-axis, respectively. All the other symbols possess their usual meanings. Following Ref. 32, Eqs. (1a-1c) can be simplified by $(i)$ setting $\phi_{M}=\phi_{H}$ (valid in high magnetic fields), and (ii) assuming no uniaxial anisotropy, i.e., $K_{U}=0$. Figure 7 shows the fitted FMR results versus angle of applied field at $16 \mathrm{GHz}$, for both $\mathrm{CoFe}$ and the Fe seed layer. In doing so, we have assumed no coupling between the FeCo and Fe seed layers (see discussion in Ref. 32). The parameters for the CoFe film were found to be $\gamma=29.4 \mathrm{GHzT}^{-1}$, $M_{0}=1.72 \times 10^{6} \mathrm{~A} \mathrm{~m}^{-1}, \phi_{C}=45^{\circ}, K_{C}=38.0 \mathrm{~kJ} \mathrm{~m}^{-3}$. The value of the cubic anisotropy parameter $K_{C}$ lies between that found for the exchange coupled bilayer $\mathrm{Ni}_{81} \mathrm{Fe}_{19}(43.5 \mathrm{~nm}) / \mathrm{Co}_{50} \mathrm{Fe}_{50}(30 \mathrm{~nm})$ of $32 \mathrm{~kJ} \mathrm{~m}^{-3}$ [Ref. 32] and the bulk value of $\sim 45 \mathrm{~kJ} \mathrm{~m}^{-3}$ [Ref. 43]. For the $\mathrm{Fe}$ seed layer, the gyromagnetic ratio $\gamma$ and $\phi_{C}$ remain the same, while $M_{0}=1.15 \times 10^{6} \mathrm{~A} \mathrm{~m}^{-1}$ and $K_{C}=13.4 \mathrm{~kJ} \mathrm{~m}^{-3}$. These values for the Fe saturation magnetization and the cubic anisotropy constant are quite different from bulk values, but are consistent with similar thin Fe films on $\mathrm{MgO}$ given in Ref. 32 .

\section{ANGULAR MODULATION OF SRR/FMR ANTICROSSING}

It has already been demonstrated, using YIG and permalloy systems, that anticrossing occurs when the frequency of the FMR approaches that of the split ring resonance ${ }^{9,17,20}$. However, unlike YIG and permalloy, the significant in-plane anisotropy of the CoFe means that the crossover field, $B_{\text {со }}$ (that field at which the FMR frequency equals that of a given SRR resonance), is now highly angle-dependent. This feature is illustrated in Fig. 8(a,b) where the transmission parameter $S_{21}^{N}\left(\nu, B_{a}\right)$ can be seen for magnetic fields applied along the easy and hard directions, respectively. For example, for the $\mathrm{TE}_{0}^{1}$ resonance at $13 \mathrm{GHz}, \mathrm{FMR}$ anticrossing occurs at $\pm 0.13 \mathrm{~T}( \pm 0.043 \mathrm{~T})$ for fields applied along the easy (hard) axis, respectively. Thus by simply rotating the 
(a)

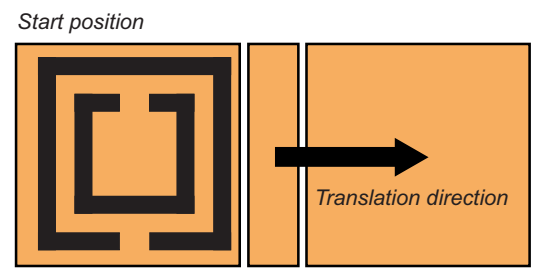

(b)

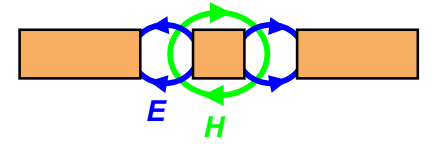

(c)

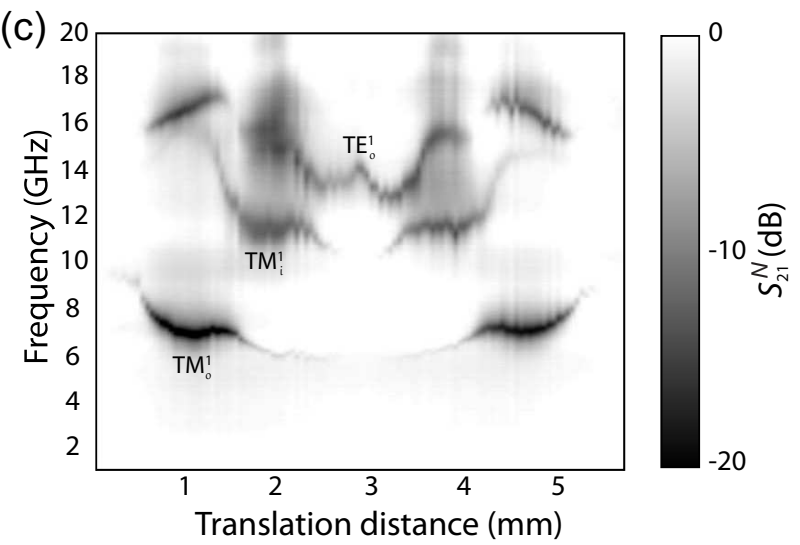

FIG. 3. (a) Top and (b) side view representations of the translation experiment, with SRR at position $0 \mathrm{~mm}$. (c) Map of $S_{21}^{N}$ versus frequency and translation, in symmetric orientation. Three modes are clearly observable which wax, wane and shift as the SRR is moved across the CPW.

magnetic field, the anticrossing for the $\mathrm{TE}_{0}^{1}$ mode can be shifted between by $\sim 0.1 \mathrm{~T}$.

There are other features apparent in Fig. 8(a,b). For example, there is an additional anticrossing with the $\mathrm{TM}_{o}^{1}$ mode at $4.0 \mathrm{GHz}$ in Fig. 8(a). However this anticrossing is absent in Fig. 8(b). The latter is forbidden, because the FMR frequency cannot drop below $8 \mathrm{GHz}$. Clearly this particular anticrossing can be switched on and off simply by rotating the magnetic field through a few degrees. Also the $\mathrm{TM}_{i}^{1}$ mode is weakly excited at $\sim 10 \mathrm{GHz}$, and reveals anticrossing with the FMR. Finally, the faint FMR-branch on the high field side of the main magnetic resonance is that generated by the Fe seed layer. This too displays anticrossing behavior, despite being $(i)$ only $3 \mathrm{~nm}$ thick and (ii) shielded by the CoFe layer, which lies between it and the CPW.

It is possible to fit the data of Fig. 8(a,b), using a simple extension to the two-level oscillator model used to describe anticrossing behavior between a SRR and FMR, in a soft magnetic material ${ }^{9,17,20}$. Given two principle SRR modes it is necessary to use a four-level coupled oscillator model. We write: (a)

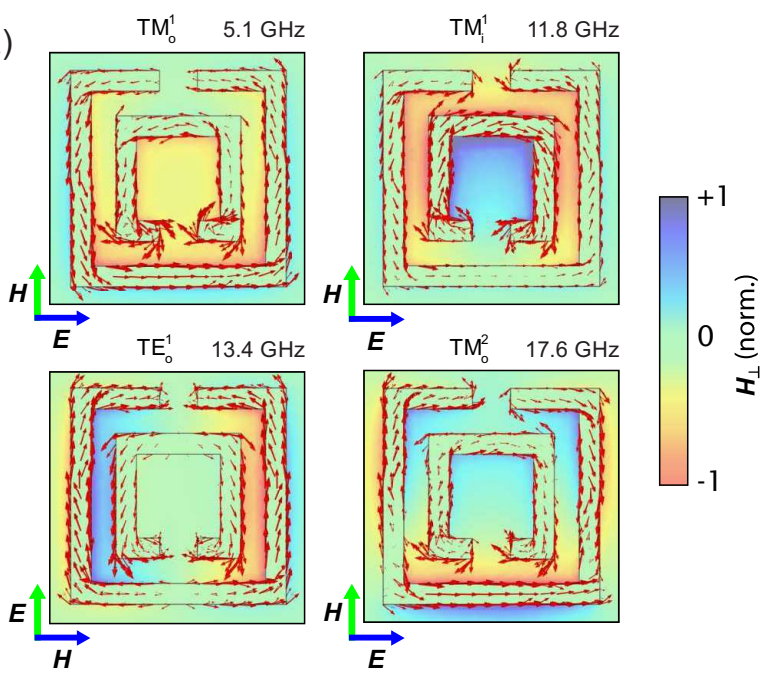

(b)

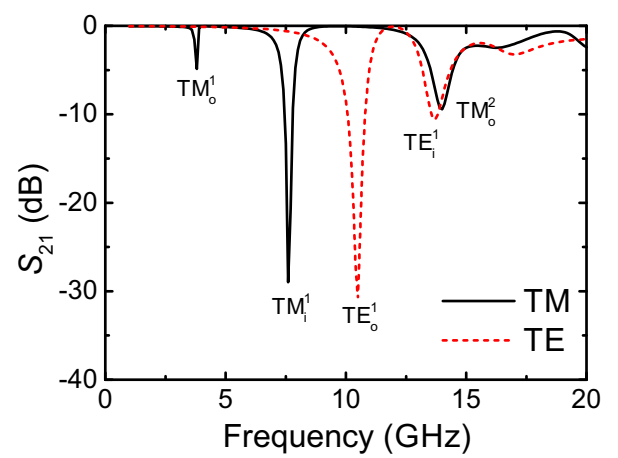

FIG. 4. (a) Computer generated snapshots: the red arrows indicate currents, while the color shading shows the out-ofplane magnetic field. (b) The scattering parameter $S_{21}$ versus frequency. This data was used to label the SRR resonances observed in Fig. 3.

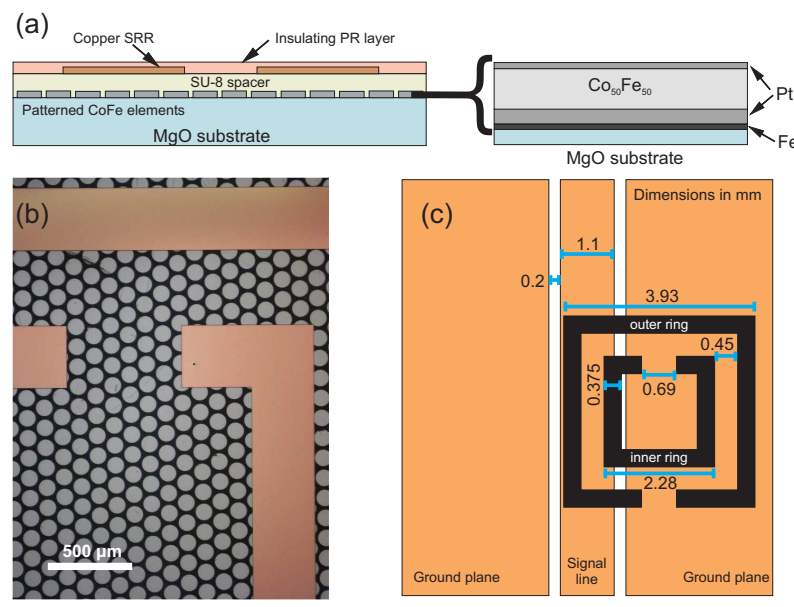

FIG. 5. (a) Schematic sample cross section of the multilayer sample showing the SRR and the patterned CoFe disks (not to scale). (b) Image of sample through an optical microscope. (c) Placement of the SRR with respect to the CPW. 


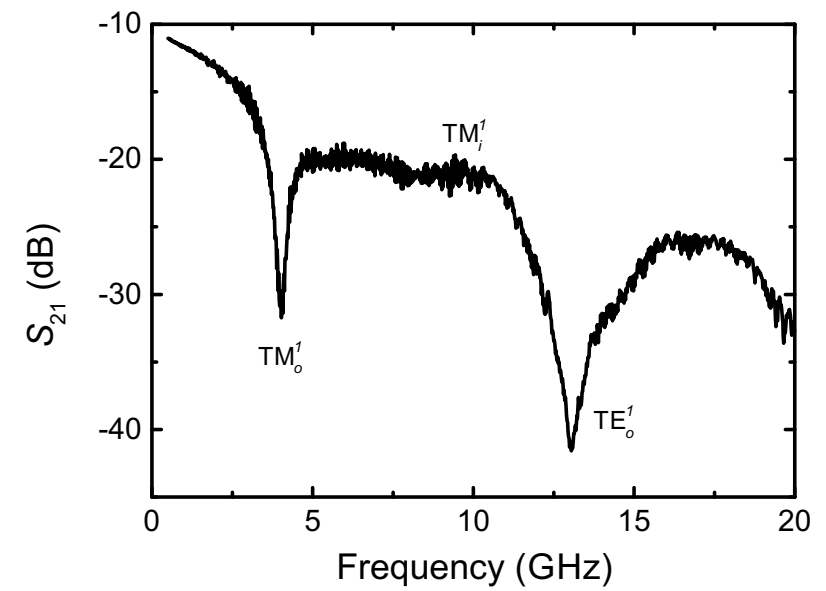

FIG. 6. The resonances of the SRR on the CPW at $B_{a}=0 \mathrm{~T}$. The chosen geometry favors two modes, $\mathrm{TM}_{o}^{1}$ at $4.03 \mathrm{GHz}$ and $\mathrm{TE}_{0}^{1}$ at $13.04 \mathrm{GHz}$. The $\mathrm{TM}_{i}^{1}$ mode is also weakly excited, but can only just be seen on this scale.

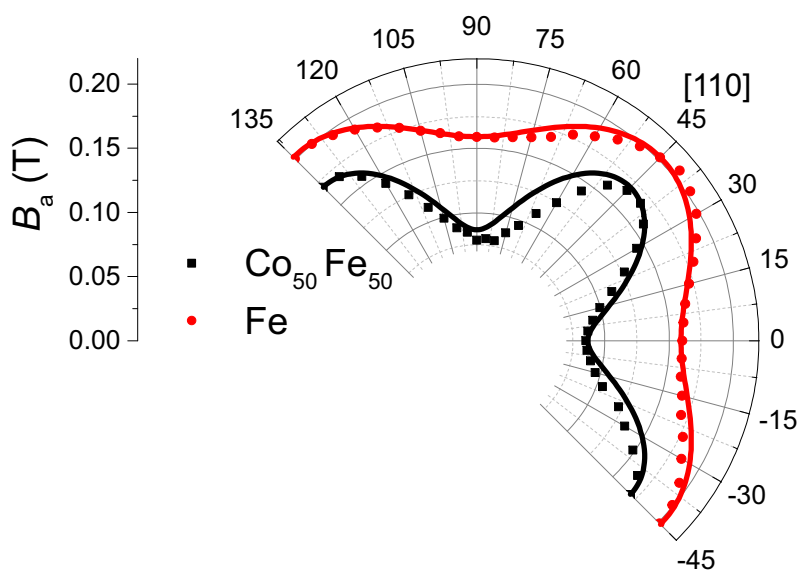

FIG. 7. FMR peak positions for the FeCo (black) and Fe-seed (red) as a function of applied field direction. The experimental FMR peak positions were determined by fitting Lorentz curves to the data. The solid lines are fits using Eq. (1). The Fe signal from the seed layer $(3 \mathrm{~nm})$ is very weak.

$$
\begin{aligned}
& \nu_{1}^{ \pm}=\frac{\nu_{\mathrm{FMR}}+\nu_{\mathrm{SRR}, 1}}{2} \pm \sqrt{\left(\frac{\left.\nu_{\mathrm{FMR}}-\nu_{\mathrm{SRR}, 1}\right)^{2}+\Delta_{1}^{2}}{2}\right.} \\
& \nu_{2}^{ \pm}=\frac{\nu_{1}^{+}+\nu_{\mathrm{SRR}, 2}}{2} \pm \sqrt{\left(\frac{\nu_{1}^{+}-\nu_{\mathrm{SRR}, 2}}{2}\right)^{2}+\Delta_{2}^{2}}
\end{aligned}
$$

Note that the $\nu_{1}^{+}$branch is essentially the FMR line sweeping up to meet the second SRR resonance.

The dashed lines in Fig. 8(a,b) represent a fit to the experimental data, obtained using Eqs. (1a) and (2b). The SRR resonance and splitting parameters were found to be $\nu_{\mathrm{SRR}, 1}=4.0 \mathrm{GHz}$ and $\nu_{\mathrm{SRR}, 2}=13.0 \mathrm{GHz}$, in
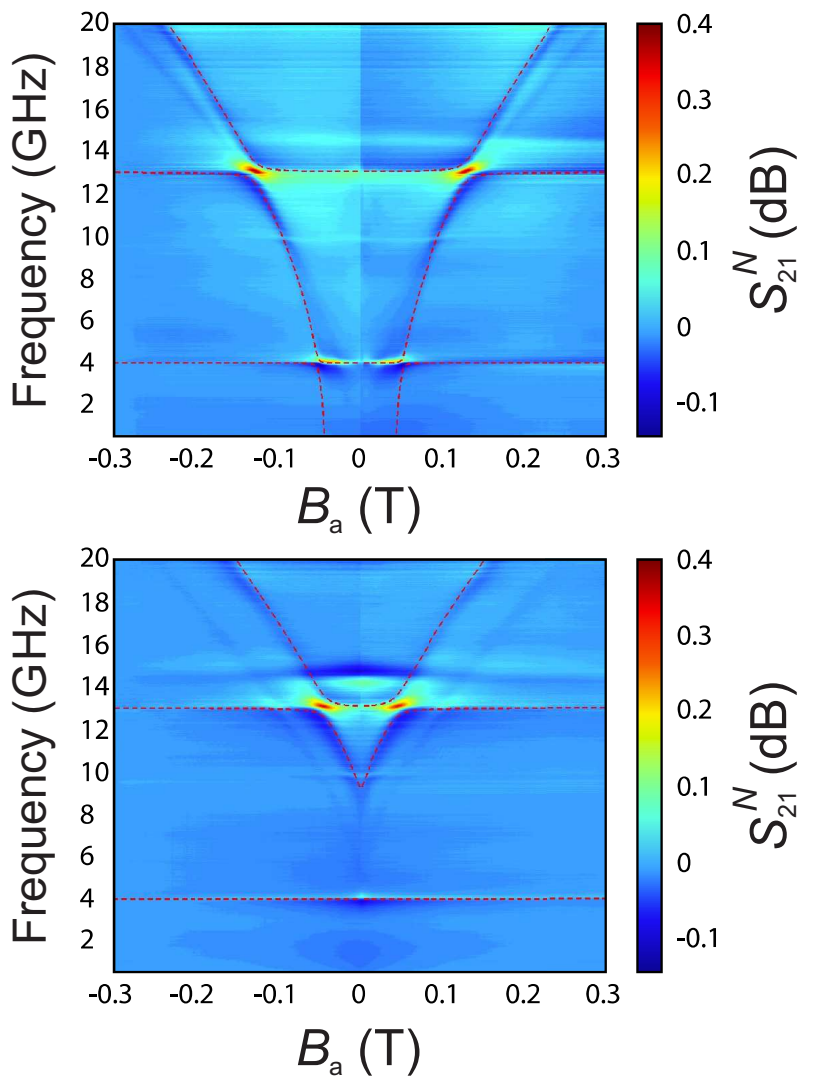

FIG. 8. Map of $S_{21}^{N}\left(\nu, B_{a}\right)$ for frequency vs. magnetic field, with the magnetic field applied along (a) easy axis $\left(0^{\circ}\right)$ and (b) hard axis $\left(45^{\circ}\right)$ of the CoFe. The raw $S_{21}$ data has been 'normalized' to a scan taken at $0.3 \mathrm{~T}$, where the FMR is beyond the available frequency range. Dashed lines are fit using a four-level coupled oscillator model.

agreement with the results presented in Fig. 6. The mutual SRR/FMR interaction parameters were found to be $\Delta_{\mathrm{SRR}, 1}=0.2 \mathrm{GHz}$ and $\Delta_{\mathrm{SRR}, 2}=0.4 \mathrm{GHz}$. These values for $\Delta$ are very similar to those of Ref. 20. However, it should be noted that the model does not take into account (i) the linewidth of the resonances involved, and (ii) broadening due to the formation of magnetic domains, particularly at low magnetic fields.

Finally, the full angular switching between the easy and hard magnetization directions can be seen in Fig. 9. At intermediate angles, the amount of modulation of the split ring resonance is reduced by $\sim 25 \%$, due to rotation of the FeCo magnetization away from a preferred axis.

\section{RESULTS FOR AN ASYMMETRIC ORIENTATION OF SRR TO CPW}

In addition to the $S_{21}^{N}\left(\nu, B_{a}\right)$ maps described in Sec. $\mathrm{V}$, many more were obtained both for differing positions and rotations of the SRR with respect to the CPW, and for patterned permalloy films. One such result, for the patterned permalloy film, can be seen in Fig. 10. Here 


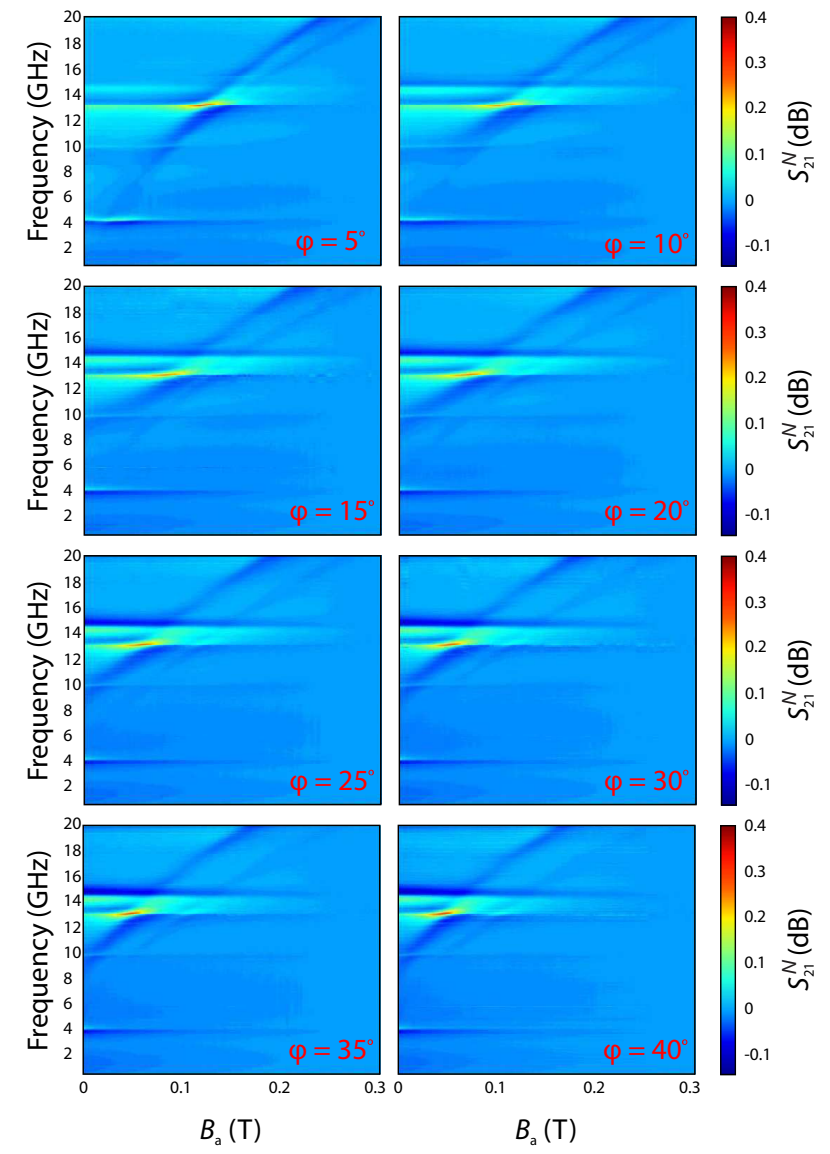

FIG. 9. Maps of $S_{21}^{N}\left(\nu, B_{a}\right)$ obtained at $5^{\circ}$ steps between the easy and hard axes.

the SRR was rotated by $90^{\circ}$ and placed at a displacement of $3.75 \mathrm{~mm}$ with respect to the center of the CPW. Both sample geometries were identical, except for the replacement of the of $\mathrm{FeCo}$ with of permalloy ${ }^{20}$.

Figure 10 can be used to illustrate two points. The first point to note is the differences in the strength of the $S_{21}^{N}$ signal between the FeCo (Figs. 8 and 9) and that obtained with permalloy (Fig. 10). The signal obtained using permalloy is some ten times stronger that of the FeCo. Clearly the mutual interaction between the SRR/FMR combination is much stronger when high permeability permalloy is used. Some of the difference can probably be attributed to the difference in thickness of the two films in question $\left(\mathrm{Ni}_{81} \mathrm{Fe}_{19}\right.$ 150-nm-thick, $\mathrm{Fe}_{50} \mathrm{Co}_{50}$ 30-nm-thick). However, one way of increasing the mutual interaction between the SRR and the FeCo film would be to decrease the distance of SU-8 insulating layer below $8 \mu \mathrm{m}$. Secondly, the identification of the various SRR modes was made in the same way as that described in Sec. III and will not be detailed here. What we wish to highlight is how well the extended multicoupled model oscillator can be used to describe multiple anticrossings. A fit to the data, using an eight-level coupled oscillator model, is represented by the red-dotted lines in Fig. 10 . The model parameters obtained in this way are listed in
Table I.

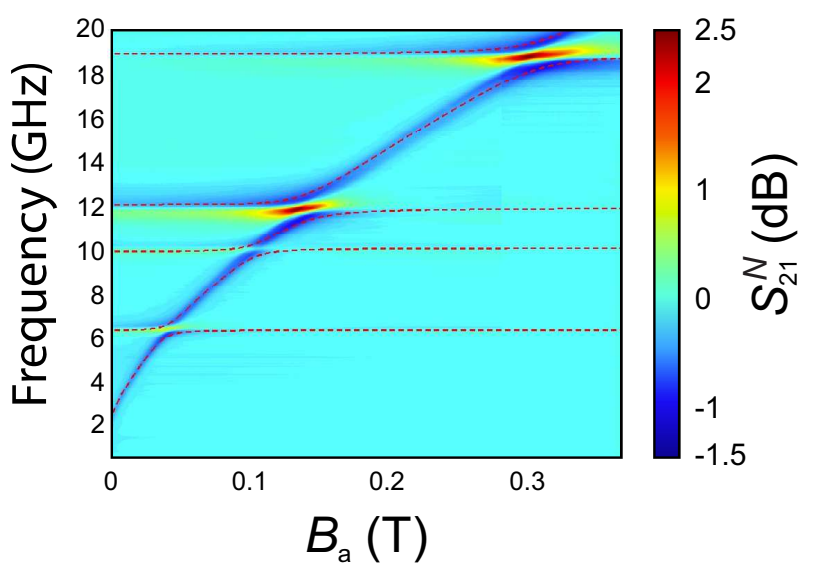

FIG. 10. Map of $S_{21}^{N}\left(\nu, B_{a}\right)$ showing four distinct SRR/FMR anticrossings, this time obtained using patterned permalloy disks. The red dashed lines represent a fit to the data using an eight-level coupled oscillator model.

\begin{tabular}{cccc}
\hline \hline $\begin{array}{c}\nu_{\mathrm{SRR}} \\
(\mathrm{GHz})\end{array}$ & Mode & $\begin{array}{c}\Delta \\
(\mathrm{GHz})\end{array}$ & $\begin{array}{c}\text { Coupling strength } \\
\left(\Delta / \nu_{\mathrm{SRR}}\right)\end{array}$ \\
\hline 6.7 & $\mathrm{TM}_{o}^{1}$ & 0.3 & $4.5 \%$ \\
10.3 & $\mathrm{TE}_{o}^{1}$ & 0.4 & $3.9 \%$ \\
12.1 & $\mathrm{TM}_{i}^{1}$ & 0.62 & $5.1 \%$ \\
18.9 & $\mathrm{TM}_{o}^{2}$ & 0.69 & $3.65 \%$ \\
\hline \hline
\end{tabular}

TABLE I. The parameters obtained using an eight-level coupled oscillator model fit to the data shown in Fig. 10. The separation at a given anticrossing is $2 \Delta$.

It is clear that the fit to the observed $\mathrm{S}_{21}^{N}\left(\nu, B_{a}\right)$ data is excellent, allowing measurements of the strength of the coupling of the SRR/FMR coupled modes. In particular, it is the $\mathrm{TM}^{1}$ modes which give the highest relative splitting $k=\Delta / \nu_{\mathrm{SRR}}$, as found by Ref. 17 . Finally, the model yields details of the hybridization of the SRR modes with FMR, at every frequency and field in the $\mathrm{S}_{21}^{N}\left(\nu, B_{a}\right)$ map. Of course, at the precise center of an anticrossing, the upper and lower modes are characterized by $50 \%$ SRR and 50\% FMR. Such mode mixing may well have applications, for coherent information transfer in microwave quantum devices which exploit strong coupling between magnons and microwave photons, as discussed for example in Refs. 17 and 44.

\section{DISCUSSION AND CONCLUSIONS}

Results have been presented for a magnetic hybridmetamolecule, involving a SRR placed in close proximity to magnetically hard thin FeCo film. To suppress eddy-current shielding, the FeCo film was patterned into $100 \mu \mathrm{m}$ disks. Two new features have been demon- 
strated: $(i)$, hard magnetic materials enable FMR and can be performed in zero or near zero applied fields, thus partially eliminating the need for an applied field; (ii), because the FMR is anisotropic, angular control over metamolecule hybrid resonances is achieved. Simply by rotating the magnetic field, the crossover field $B_{\text {co }}$ at a given anticrossing can be varied from 0.04 to $0.13 \mathrm{~T}$. This degree of control is much larger than that which can be obtained by sweeping across a single anticrossing ${ }^{20}$. In addition, a special study has been reported on the effect of the near-field CPW-excitation on SRR resonances. Here, both the character and strength of the signal depends strongly on the relative position of the SRR with respect to the CPW. In particular, it has been shown how to identify and optimize the various transverse magnetic (TM) and transverse electric (TE) modes of the SRR, using near-field CPW-excitation. Such detail is important, if the strongest signal/modulation of a metamolecule resonance is to be achieved.

It is also worth noting that the use of hard materials, with remanent magnetization, opens the door to magnetically reconfigurable metamaterials in the time domain. For example, the resolution of magnetic switching in hard drive technology is of order $10 \mathrm{~nm}$. Such resolution is more than enough for local switching of the FeCo magnetic disks above the SRR. So we have the possibility of manipulating the SRR/FeCo magnetic coupling in a time-sequential fashion. For example, the near field maps of Fig. 4(a) show the simulated out-of-plane magnetic field components for the first four modes of the SRR. These fields compete with the out-of-plane component of the FMR, and it this competition which gives rise to anticrossing behavior. However, in a magnetically configurable metamaterial, the magnetization of the $\mathrm{FeCo}$ disks can be realigned allowing the SRR/FMR coupling to be modified in the time-domain. For example, in the $\mathrm{TM}_{i}^{1}$ mode at $11.8 \mathrm{GHz}$ [see Fig. 4(a) top-left] the fields in the SRR gaps are both pointing-down (both shaded orange). However in the $\mathrm{TE}_{i}^{1}$ mode at $13.4 \mathrm{GHz}$ the two fields in question are pointing in opposite directions (one shaded blue, one shaded orange). So by switching the magnetization of the FeCo disks above the two gaps, from in-plane to out-of-plane, SRR/FMR hybridization of these modes can be switched on/off.

Finally, it has been demonstrated that the simple twolevel SRR/FMR oscillator model, developed to explain a single anticrossing ${ }^{17,20}$, can be very successfully extended to deal with the numerous TM and TE modes of an SRR coupled to a ferromagnetic film.

\section{ACKNOWLEDGMENTS}

We acknowledge Diamond Light Source for time on beamline I10 under proposal SI-10152. SAG gratefully acknowledges funding by the Defence and Security PhD scheme managed by Dstl.
1 D. R. Smith, J. B. Pendry, and M. C. K. Wiltshire, "Metamaterials and negative refractive index," Science 305, 788792 (2004).

2 T. Xu, A. Agrawal, M. Abashin, K. J. Chau, and H. J. Lezec, "All-angle negative refraction and active flat lensing of ultra-violet light," Nature 497, 470-474 (2013).

3 N. I. Zheludev and Y. S. Kivshar, "From metamaterials to metadevices," Nat. Mater. 11, 917-924 (2012).

${ }^{4}$ K. Tanaka, E. Plum, J. Y. Ou, T. Uchino, and N. I. Zheludev, "Multifold enhancement of quantum dot luminescence in plasmonic metamaterials," Phys. Rev. Lett. 105, 227403 (2010).

5 O. Hess, J. B. Pendry, S. A. Maier, R. F. Oulton, J. M. Hamm, and K. L. Tsakmakidis, "Active nanoplasmonic metamaterials," Nat. Mater. 11, 573-584 (2012).

6 J. Zhou, D. R. Chowdhury, R. Zhao, A. K. Azad, H.-T. Chen, C. M. Soukoulis, A. J. Taylor, and J. F. O'Hara, "Terahertz chiral metamaterials with giant and dynamically tunable optical activity," Phys. Rev. B 86, 035448 (2012).

7 S. Zhang, J. Zhou, Y.-S. Park, J. Rho, R. Singh, S. Nam, A. K. Azad, H.-T. Chen, X. Yin, A. J. Taylor, and $\mathrm{X}$. Zhang, "Photoinduced handedness switching in terahertz chiral metamolecules," Nat. Commun. 3, 942 (2012).

8 Y.-J. Yang, Y.-J. Huang, G.-J. Wen, J.-P. Zhong, H.-B. Sun, and O. Gordon, "Tunable broadband metamaterial absorber consisting of ferrite slabs and a copper wire," Chin. Phys. B 21, 038501 (2012).
${ }^{9}$ G. B. G. Stenning, G. J. Bowden, L. Maple, S. A. Gregory, A. Sposito, R. W. Eason, N. I. Zheludev, and P. A. J. de Groot, "Magnetic control of a meta-molecule," Opt. Express 21, 1456-1464 (2013).

10 L. Kang, Q. Zhao, H. Zhao, and J. Zhou, "Magnetically tunable negative permeability metamaterial composed by split ring resonators and ferrite rods," Opt. Express 16, 8825-8835 (2008).

11 Y. J. Huang, G. J. Wen, Y. J. Yang, and K. Xie, "Tunable dual-band ferrite-based metamaterials with dual negative refractions," Appl. Phys. A 106, 79-86 (2012).

${ }^{12}$ H. Zhao, J. Zhou, Q. Zhao, B. Li, L. Kang, and Y. Bai, "Magnetotunable left-handed material consisting of yttrium iron garnet slab and metallic wires," Appl. Phys. Lett. 23, 131107 (2007).

13 J. N. Gollub, D. R. Smith, and J. D. Baena, "Hybrid resonant phenomenon in a metamaterial structure with integrated resonant magnetic material," Opt. Express 17, 5-?? (2008).

14 K. Bi, J. Zhou, H. Zhao, X. Liu, and C. Lan, "Tunable dual-band negative refractive index in ferrite-based metamaterials," Opt. Express 9, 10746-10752 (2013).

15 J. Ghalibafan, N. Komjani, and B. Rejaei, "Design and analysis of a novel tunable ferrite based left handed strip line," J. Electromagnet. Wave. 7, 914-922 (2012).

16 Y. Huang, G. Wen, T. Li, and K. Xie, "Lowloss, broadband and tunable negative refractive index metamaterial," JEMAA 2, 104-110 (2010). 
17 B. Bhoi, T. Cliff, I. S. Maksymov, M. Kostylev, R. Aiyar, N. Venkataramani, S. Prasad, and R. L. Stamps, "Study of photon-magnon coupling in a YIG-film split-ring resonant system," J. Appl. Phys. 116, 243906 (2014).

18 G. P. Rodrigue, "A generation of microwave ferrite devices," Proc. IEEE 76, 121-137 (1988).

19 V. G. Harris, A. Geiler, Y. Chen, S. D. Yoon, M. Wu, A. Yang, Z. Chen, P. He, P. V. Parimi, X. Zuo, C. E. Patton, M. Abe, O. Acher, and C. Vittoria, "Recent advances in processing and applications of microwave ferrites," J. Magn. Magn. Mater. 321, 2035-2047 (2009).

20 S. A. Gregory, G. B. G. Stenning, G. J. Bowden, N. I. Zheludev, and P. A. J. de Groot, "Giant magnetic modulation of a planar, hybrid metamolecule resonance," New J. Phys. 16, 063002 (2014).

21 F. Martin, F. Falcone, J. Bonache, R. Marques, and M. Sorolla, "Miniaturized coplanar waveguide stop band filters based on multiple tuned split ring resonators," IEEE Microw. Compon. Lett. 13, 511-513 (2003).

22 F. Martin, J. Bonache, F. Falcone, M. Sorolla, and R. Marques, "Split ring resonatorbased left-handed coplanar waveguide," Appl. Phys. Lett. 83, 4652 (2003).

${ }^{23}$ F. Falcone, F. Martin, J. Bonache, R. Marques, and M. Sorolla, "Coplanar waveguide structures loaded with split-ring resonators," Microw. Opt. Techn. Let. 40, 3-6 (2004).

${ }^{24}$ I. Gil, J. Bonache, J. Garcia-Garcia, and F. Martin, "Tunable metamaterial transmission lines based on varactorloaded split-ring resonators," IEEE T. Microw. Theory 54, 2665-2674 (2006).

${ }^{25}$ M. Geissler and Y. Xia, "Patterning: Principles and some new developments," Adv. Mater. 16, 1249 (2004).

26 J. Stöhr and H. C. Siegmann, "Magnetism," (2006).

27 F. Pulizzi, "Spintronics," Nat. Mater. 11, 367 (2012).

${ }^{28}$ V. Novosad, F. Y. Fradin, P. E. Roy, K. S. Buchanan, K. Yu. Guslienko, and S. D. Bader, "Magnetic vortex resonance in patterned ferromagnetic dots," Phys. Rev. B 72, 024455 (2005).

29 A. Fert, V. Cros, and J. Sampaio, "Skrymions on the track," Nat. Nano. 8, 152-156 (2013).

30 B. Lenk, H. Ulrichs, F. Garbs, and M. Münzenberg, "The building blocks of magnonics," Phys. Rep. 507, 107-136 (2011).

31 V. V. Kruglyak, S. O. Demokritov, and D. Grundler, "Magnonics," J. Phys. D: Appl. Phys. 43, 264001 (2010).

${ }^{32}$ G. B. G. Stenning, L. R. Shelford, S. A. Cavill, F. Hoffmann, M. Haertinger, T. Hesjedal, G. Woltersdorf, G. J.
Bowden, S. A. Gregory, C. H. Back, P. A. J. de Groot, and G. van der Laan, "Magnetization dynamics in an exchangecoupled $\mathrm{NiFe} / \mathrm{FeCo}$ bilayer studied by x-ray detected ferromagnetic resonance," New J. Phys. 17, 013019 (2015).

33 "MicroChem SU-8 Permanent Epoxy Negative Photoresist," http://www . microchem.com/Prod-SU83000.htm, accessed: 2015-08-29.

${ }^{34}$ G. van der Laan and A. I. Figueroa, "X-ray magnetic circular dichroism - A versatile tool to study magnetism," Coord. Chem. Rev. 277-278, 95-129 (2014).

35 R. N. Simons, Coplanar Waveguide Circuits, Components E Systems (John Wiley \& Sons, 2004).

36 J. D. Baena, J. Bonache, F. Martin, R. M. Sillero, F. Falcone, T. Lopetegi, M. A. G. Laso, J. Garcia-Garcia, I. Gil, M. F. Portillo, and M. Sorolla, "Equivalent-circuit models for split-ring resonators and complementary split-ring resonators coupled to planar transmission lines," IEEE T. Microw. Theory 53, 1451-1461 (2005).

37 J. Zhou, T. Koschny, and C. M. Soukoulis, "Magnetic and electric excitations in split ring resonators," Opt. Express 15, 17881-17890 (2007).

38 N. Liu, H. Guo, L. Fu, H. Schweizer, S. Kaiser, and H. Giessen, "Electromagnetic resonances in single and double split-ring resonator metamaterials in the near infrared spectral region," Phys. Status Solidi B 244, 1251-1255 (2007).

39 H. Guo, N. Liu, L. Fu, T. P. Meyrath, T. Zentgraf, H. Schweizer, and H. Giessen, "Resonance hybridization in double split-ring resonator metamaterials," Opt. Express 15, 12095-12101 (2007).

40 "Glass-reinforced epoxy laminate sheets," http://www. farnell.com/datasheets/1644697.pdf, accessed: 201508-29.

41 C. Kittel, "On the theory of ferromagnetic resonance absorption," Phys. Rev. 460, 155-161 (1948).

42 M. Farle, "Ferromagnetic resonance of ultrathin metallic layers," Rep. Prog. Phys. 61, 755-826 (1999).

43 T. Kuschel, J. Hamrle, J. Pistora, K. Saito, S. Bosu, Y. Sakuraba, K Takanashi, and J. Wollschläger, "Magnetic characterization of thin $\mathrm{Co}_{50} \mathrm{Fe}_{50}$ films by magnetooptic Kerr effect," J. Phys. D: Appl. Phys. 45, 495002 (2012).

44 H. Huebl, C. W. Zollitsch, J. Lotze, F. Hocke, M. Greifenstein, A. Marx, R. Gross, and S. T. B. Goennenwein, "High coperativity in coupled microwave resonator ferrimagnetic insulator hybrids," Phys. Rev. Lett. 111, 127003 (2013). 